\title{
Mobile APPLiCATION FOR SKIN CANCER RisK PREDICTION BASED ON EMBEDDED CONVOLUTION NEURAL NETWORK MODEL
}

\author{
Bruno Trstenjak \& Marko Hrncic
}
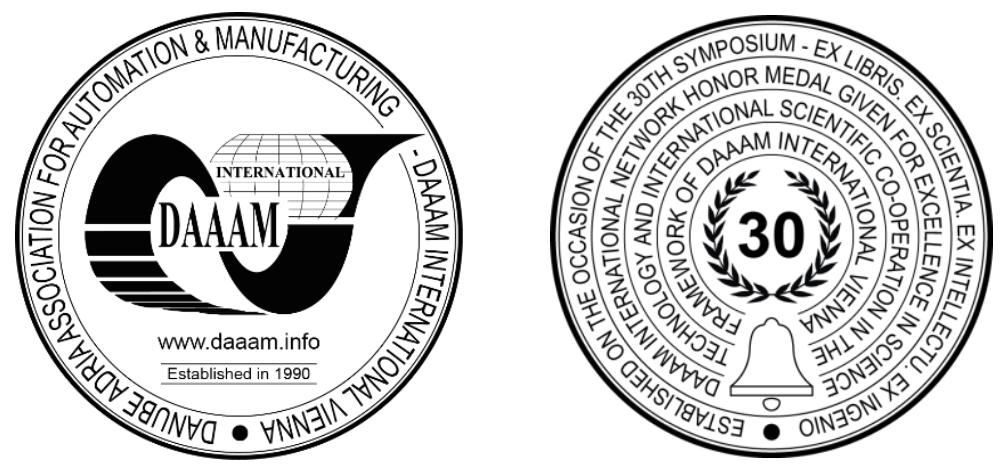

This Publication has to be referred as: Trstenjak, B[runo] \& Hrncic, M[arko] (2020). Mobile Application for Skin Cancer Risk Prediction Based on Embedded Convolution Neural Network Model, Proceedings of the 31st DAAAM International Symposium, pp.0209-0217, B. Katalinic (Ed.), Published by DAAAM International, ISBN 978-3-90273429-7, ISSN 1726-9679, Vienna, Austria

DOI: $10.2507 / 31$ st.daaam.proceedings.028

\begin{abstract}
The skin cancer has been a serious problem globally for a long time. For the prevention and early detection of this type of cancer, various scientific research and applications have been published. This paper is addressed to the introduction of a modern mobile application for the skin cancer risk prediction based on the embedded Convolution Neural Network model. The paper explains the working principle of the application, the structure of the model and how it is embedded in the mobile application. The results of testing and measuring the prediction accuracy are presented compared with similar classification models published in several scientific studies. The mobile app is downloaded and used by users from all over the world. In this study, the analysis of actual prediction results was performed, the results of risk assessment and geographical distribution of the users were actually measured. The end of the article presented the guidelines for further research and development of the prediction model.
\end{abstract}

Keywords: Convolution Neural Network; health; melanomas; mobile application; skin cancer.

\section{Introduction}

The skin cancer has been a serious problem globally for a long time and the incidence of both non-melanoma and melanoma skin cancers has been increasing over the past decade. The data collected globally show that between 2 and 3 million non-melanoma skin cancers and 132,000 melanoma skin cancers occur globally each year [1].

On the basis of such information, the early prevention and detection of skin cancer has become a high-ranking task of the scientific research community. This fact is supported by a lot of scientific research published over the past decade, which proposed various methods and approaches to solving the problems of early prevention. Machine learning and deep learning is revolutionizing the medical field by helping to analyse the images and collecting data in order to detect various diseases. For the purposes of image analysis, computer vision and pattern recognition [2], the convolutional neural networks method has been imposed as a method implemented in various algorithms for medical purposes. 
A number of papers are presented in image classification using deep convolutional neural networks, such as Alzheimer's disease classification from magnetic resonance brain images [3], detection and classification of breast cancer [4], using CNN models for accurate brain tumour segmentation [5], CNN for bone age assessment [6], brain image analysis on magnetic resonance imaging [7] etc.

One of the possible approaches to achieving the early prevention and detection of skin cancer is to give people the ability to independently test their skin. Nowadays more and more people use smartphones equipped with quality cameras, so one of the possible solutions imposed would be a mobile application for skin cancer risk assessment. The research we conducted was focused on the development of the classification module for risk prediction of skin cancer, a prediction model suitable to be embedded into a modern mobile application. In this paper, we focused to present a CNN based prediction model, the mobile application structure description and the prediction accuracy results achieved with the embedded model.

The rest of the paper is organized as follows. Section II briefly presents some related work, different research of the concepts of classification models in skin cancer recognition using different machine learning techniques. In the Section III, we introduce our methodology for the creation of embedded CNN model. Section IV describes the design and features of the proposed application. The achieved experimental results of the case study are explained in Section V. Finally, Section. VI gives the conclusion of the paper and provides future directions.

\section{Literature review}

In the domain of the prevention and early detection of this type of cancer, many, various scientific research and papers which propose different approaches and methods in skin cancer recognition have been published. Today, when we are witnessing technological progress, it is becoming commonplace for smartphones to be used for a variety of needs. They are also used for medical purposes. Cameras built into mobile devices allow users to take pictures of parts of their body and independent of the laboratory using Artificial Intelligence perform their analysis. These facts make smartphone to become an ideal tool for early prevention of skin cancer. In many phones intelligent systems for image analysis are already embedded. With the use of Artificial Neural Networks and various algorithms, smartphones conduct the skin images analysis in order to identify skin cancer [8].

Many published studies have used the CNN network for image recognition. In a recently published study for the purpose of identifying melanoma CNN networks were used in such a way that CNN was trained using dermoscopic images. The study performed measurements over the CNN model and the accuracy degree in identifying melanoma used clinical photographs with the manual grading of the same images by dermatologists [9].

In order to improve the performance of the $\mathrm{CNN}$, various hybrid classification methods have also been developed. A fully automatic computerised method to classify skin lesions from dermoscopic images is presented. It is presented on a novel ensemble scheme for convolutional neural networks (CNNs) that combines intra-architecture and inter-architecture network fusion. The proposed method consists of multiple sets of CNNs of different architecture that represent different feature abstraction levels [10].

For the purpose of classifying melanoma images the research was published in which it was investigated how to create an ensemble of deep convolutional neural networks to further improve their individual accuracies in the task of classifying dermoscopy images into three classes melanoma, nevus and seborrheic keratosis when there is no opportunity to train them on adequate number of annotated images [11]. As the skin is constantly exposed to sunlight, it has been presented an innovative and fully functional smartphone application to assist in melanoma early detection and prevention. The developed application allows a real-time alert to help users prevent skin burn caused by sunlight and automated image analysis with hair detection and exclusion, lesion segmentation, feature extraction, and image classification [12].

A somewhat different approach to early prevention of skin cancer has been shown in research in which a mobile melanoma detection application which could be used for the identification of melanoma on the skin in its earliest stages was developed. The application uses colour and symmetry based analysis with a Support Vector Machine (SVM) to classify the image as benign or malignant. Results of skin cancer detection are sent back by the system to the user and assist in the process to seek professional services [13].

\section{Research methodology and the proposed framework}

The study was conducted with the aim to develop a mobile application that will achieve a high degree of skin cancer risk prediction accuracy. A developed mobile application uses an embedded prediction model in its operation without the need to send images to a remote internet platform. In the development of the mobile application, there were the following demands: the quality and high accuracy of the prediction do not depend on the type of mobile device, the application enables prediction over photos of different dimensions and resolutions, possibility of fast prediction and presentation of results, the ability to send prediction results to the cloud platform for further research.

By studying the models which have been published in various scientific studies, comparing their positive and negative features, we decided for the basic method for risk prediction to be the Convolutional Neural Network (CNN). In recent times, the Convolutional Neural Networks have become the most powerful method for image classification. 
Various researchers have shown the importance of network architecture in achieving better performances by making changes in different layers of the network [14].

When taking into consideration all the requirements and if the prediction takes place at the mobile device level, for the development of the prediction model Tensor Flaw MobileNetV2 library was selected. The MobileNetV2 is an architecture proposed by Google which is suitable for mobile and embedded based vision applications [15].

\subsection{Pre-processing}

Due to the use of different mobile devices, the input images of mole or parts of the skin may have different properties. For this reason, before the process of prediction, it is necessary to make image adjustment. The pre-processing is the process of preparing input data before the data is sent to the classification model.

The pre-processing allows to solve the problem of a noisy or distorted image dataset, adjusting image features that are appropriate for CNN input features [16]. For mobile application purposes, all input images are adjusted and resized to $224^{2} \times 3$ size. After resizing the image, before a risk prediction starts, a mobile application starts image conversion and adjustment to mobilenet_v2 predictive model features.

\subsection{Embedded CNN}

Convolutional neural network is an effective machine learning technique from deep learning and it is similar to ordinary Neural Networks. Convolutional neural network is a network with convolutional layers. Convolutional neural network consists of three steps of neural layers to build its architectures: Convolutional, Pooling, and Fully-Connected [17].

The MobileNetV2 architecture is based on an inverted residual structure, uses lightweight depthwise convolutions to filter features in the intermediate expansion layer. The architecture of MobileNetV2 contains the initial fully convolution layer with 32 filters, followed by 19 residual bottleneck layers, as shown in Table 1.

\begin{tabular}{|c|c|c|c|c|c|}
\hline Input & Operator & t & c & n & s \\
\hline $224^{2} \times 3$ & conv2d & - & 32 & 1 & 1 \\
\hline $112^{2} \times 32$ & bottleneck & 1 & 16 & 1 & 1 \\
\hline $112^{2} \times 16$ & bottleneck & 6 & 24 & 2 & 2 \\
\hline $56^{2} \times 24$ & bottleneck & 6 & 32 & 3 & 2 \\
\hline $28^{2} \times 32$ & bottleneck & 6 & 64 & 4 & 2 \\
\hline $14^{2} \times 64$ & bottleneck & 6 & 96 & 3 & 1 \\
\hline $14^{2} \times 96$ & bottleneck & 6 & 160 & 3 & 2 \\
\hline $7^{2} \times 160$ & bottleneck & 6 & 320 & 1 & 1 \\
\hline $7^{2} \times 320$ & conv2d & - & 1280 & 1 & 1 \\
\hline $7^{2} \times 1280$ & avgpool & - & - & 1 & - \\
\hline $1 \times 1 \times 1280$ & conv2d 1x1 & - & $k$ & - & \\
\hline
\end{tabular}

Table 1. MobileNetV2 overall architecture

where t: expansion factor, c: number of output channels, n: repeating number, s: stride. $3 \times 3$ kernels are used for spatial convolution. Typically, the primary network (width multiplier 1, 224×224), CNN has a computational cost of 300 million multiply-adds and uses 3.4 million parameters [15]. In our research we have also used such a configuration, as a primary network.

In order to improve the prediction accuracy, we have added a few layers to Standard MobileNetV2 architecture. Additional layers ensure the fine-tuning of the classification model and fitting the model to the characteristic features of the disease. Fine-tuning is the process of taking a pre-trained model (MobileNetV2 model) and use it as the starting point for optimizing a different task. We have added a task-specific classification layer on the pre-trained model. Fig. 1 shows the proposed architecture of out mobile fine-tuning classification model. Base classification model is MobileNetV2 model set to use the input image shape size 224 x 224 with 3 channels, with a global average pooling layer. The base model uses ReLU6 activation function between the layers. Fig. 2 shows the characteristic of the ReLU6 activation function. The basic model is followed by layers for fine-tuning. 


\section{Base model}

\section{MobileNetV2}

input shape $(224,224,3)$

pooling (average)

activation (ReLU6)

batch Normalization

\section{Fine tuning}

\section{LAYER}

Top input model

Base model - output shape

\section{LAYER}

GlobalAveragePooling2D

\section{LAYER}

Reshape $(1,1,1280)$

feature inputs (Top input model)

\section{LAYER}

Dropout (0.5)

\section{LAYER}

Softmax layer

\section{LAYER}

Reshape (7) output layer

Fig. 1. Architecture of the embedded CNN

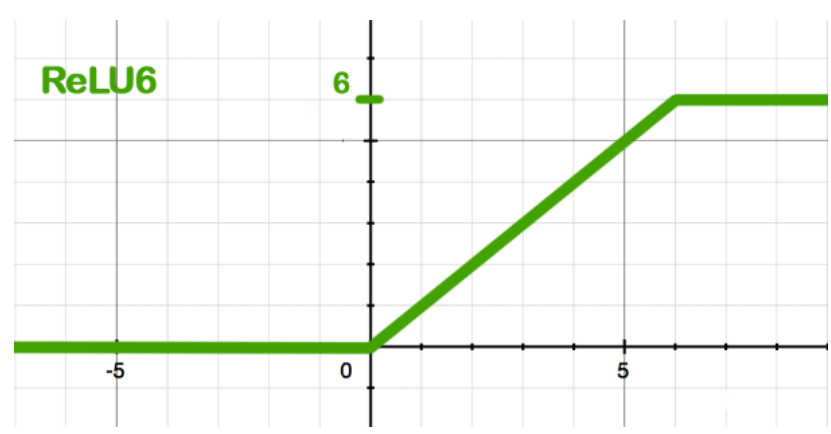

Fig. 2. ReLU6 activation function

The first layer collects the output features of the base model. After that, the data is passed to the average Pooling 2D layer to perform down-sampling by dividing the input into rectangular pooling regions and computing the average values of each region. The pooling layer follows a reshape layer which reshapes an output to a required 1 x 1 x 1280 shape. In order to reduce overfitting and improve generalization error, a dropout layer was added into the classification model. Dropout refers to ignoring of some neurons during the network training. This approach improves generalization of future CNN because force model during the training to learn with different neurons.

After the dropout layer, the additional term penalizes large weight values, all in order to reduce overfitting. The two most common error functions used in neural network training are squared error and cross entropy error. As softmax layer was used in the model, the cross entropy loss function is selected to reduce overfitting. 
The Cross Entropy Loss function is defined as:

$$
C E=-\sum_{i=1}^{C} t_{I} \log \left(f(s)_{i}\right)
$$

where $t_{i}$ and $s_{i}$ are the ground truth and the CNN score for each class $i$ in $C, f(s)_{i}$ is activation function.

At the end of the classification model the softmax and reshape layers have been added. A softmax layer applies a softmax function to the input. Function is to turn the score produced by the neural network into values within the range $[0,1]$. The assigned values present probabilities to each class in a multi-class problem. The reshaped layer based on the calculated probabilities performs the final classification of the input image.

\subsection{Technical approach and solution}

Today, in the age of smartphones, we have witnessed the appearance of a large number of mobile applications that are developed for medical purposes. The main objective of our research was development of fully functional smart-phone application to assist in skin cancer early detection. A developed smartphone application supports smart phones based on Android operating system. The application consists of three activities:

- Prediction activity - the main activity for the prediction of risk. The activity allows the user to take an image of the mole, start the prediction process and record the results and the image in the database on the mobile device.

- List activity - allows the user to view and search the results of past predictions

- Comparison activity - allows the user to compare the images, to determine if there is any difference in the appearance of moles.

Figure 3 shows the proposed architecture of the main program activity in mobile application. The main program activity for risk prediction is divided into several internal methods. The activity begins with a method which allows the user to take its own image of mole (skin). The created image is forwarded into pre-processing method to the image optimization and adjustment to start prediction. The method improves the image quality and reduces or removes image interferences.

Thus prepared image is forwarded to embedded CNN model, whose architecture and concept is shown in Fig 1. For the purposes of the mobile application, the embedded model described in the previous section is specifically adapted to run on the Android operating system. For this reason and needs, we have selected the MobileNetV2 library as the base platform in the development of our CNN model.

During the process of CNN model development, testing, and fine-tuning to achieve the highest possible classification accuracy, the final version of the $\mathrm{CNN}$ model is written in a format suitable for various transformations. After testing, the final version was transformed into a new model adapted for Android OS and embedded into the mobile application. This enables the entire skin cancer risk prediction process to be carried out at the mobile device level, without the need for internet connectivity. This approach has enabled greater speed of application and provided the users autonomy during the use of the application.

After classification of the input image, based on the likelihood results, "Class determination" method determines the class affiliation. The method calculates the level of the risk and prepare the results for presentation. Developed embedded CNN model supports image multi-class classification. The application analyses the captured image and classifies the image mole into seven classes.

\begin{tabular}{|c|l|c|}
\hline Class & \multicolumn{1}{|c|}{ Descript } & No. of Images \\
\hline 1 & AKIEC (MALIGNANT) & 326 \\
\hline 2 & BCC (MALIGNANT) & 533 \\
\hline 3 & BKL (BENIGN) & 1184 \\
\hline 4 & DF (BENIGN) & 113 \\
\hline 5 & MEL (MALIGNANT) & 1184 \\
\hline 6 & NV (BENIGN) & 5954 \\
\hline 7 & VASC (BENIGN) & 148 \\
\hline
\end{tabular}

Table 2. Image classes 


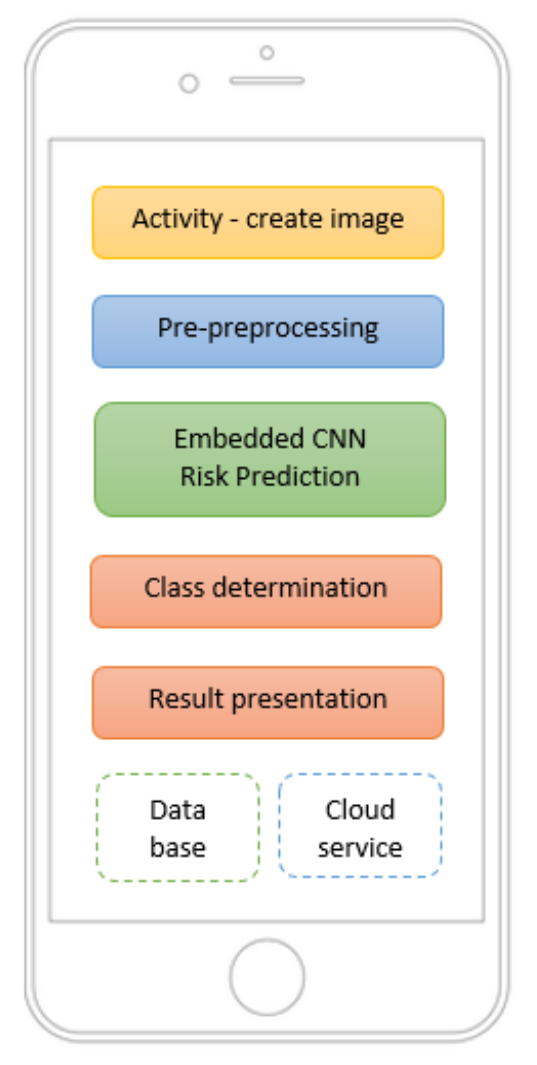

Fig. 3. Architecture of the mobile application

During developing the CNN model, we have performed image clustering. Seven clusters were formed, as shown in Table 2. We have decided to use image clustering and multi-class approach instead of binary classification because we have archived much better results and a higher classification accuracy. Four clusters were created for the images of a benign mole. Method "Result presentation" is in charge to display results of the skin cancer risk prediction and presents additional types and information to user. The method allows the user to return to the beginning of the activity and to repeat the entire prediction process. The final result of prediction and risk assessment is classified into three classes: low, middle and high risk for skin cancer. If the user thinks the prediction result is realistic, a method enables the user to save the results of a risk assessment. The prediction results are written into the relational database and the image into the smartphone local memory. Parallelly with the data saving process, the application uses the cloud service module. The service is used to send the obtained result of skin cancer prediction to a cloud framework. The cloud framework is designed to accept the prediction results of various diseases from other mobile application [18].

\section{Experimental results and discussion}

\subsection{Dataset}

In this research, we evaluate the proposed CNN model performance by using a dataset of over 9000 images. The images have been collected from the international skin imaging collaboration HAM10000 database. These images belong to two main classes, melanoma and non-melanoma. The dataset comprises of 10,015 images belonging to 7 classes, namely: MEL (Melanoma), NV (Melanocytic Nevus), BCC (Basal Cell Carcinoma), AKIEC (Actinic Keratosis), BKL (Benign Keratosis), DF (Dermatofibrosis), and VASC (Vascular Lesions), obtained from the HAM10000 dataset.

The training data distribution was highly imbalanced, which prompted us to approach this classification problem in a slightly different way. The following is an enumeration over the seven distinct target categories, along with a brief description of each category [19].

\subsection{Mobile application}

The mobile application was released in July 2019 on the Google Play platform. According to the latest statistics by Google, the application has been downloaded more than 1,500 people worldwide. Fig. 4 shows the mobile application, its activity for skin cancer prediction. Before the application was released, detailed tests were conducted with an emphasis on prediction accuracy. 
In testing, we focused on the quality of work of the classification algorithm, the influence of clustering images on the prediction accuracy of the embedded $\mathrm{CN}$ model, how image quality and type of the device influence the results of prediction. During testing, the proposed CNN model achieved a high degree of prediction accuracy. Table 3 shows the achieved prediction results of several different published studies of the classification of skin cancer and the average result achieved by our model. According to the results, it can be observed that the proposed CNN model achieved a relatively high degree of accuracy. In order to obtain complete details about the characteristics of the CNN model, additional analysis of model performances using the Receiver Operating Characteristic (ROC) curve was performed [20]. Fig. 5 shows the ROC curves, measurements of classification accuracy over a few image datasets. The shapes of the ROC curves indicate a very good performance of CNN model.

In developing and publishing applications, attention was paid to the protection and privacy of personal data. The mobile application is published under the GDPR license, use of the application is anonymous, all data and scanned images are not sent to the server but remain on the user's mobile phone. The reason is that the goal of the project was not a collection of prediction results, but the possibility of early prevention of skin cancer and warning users about the possible risk.
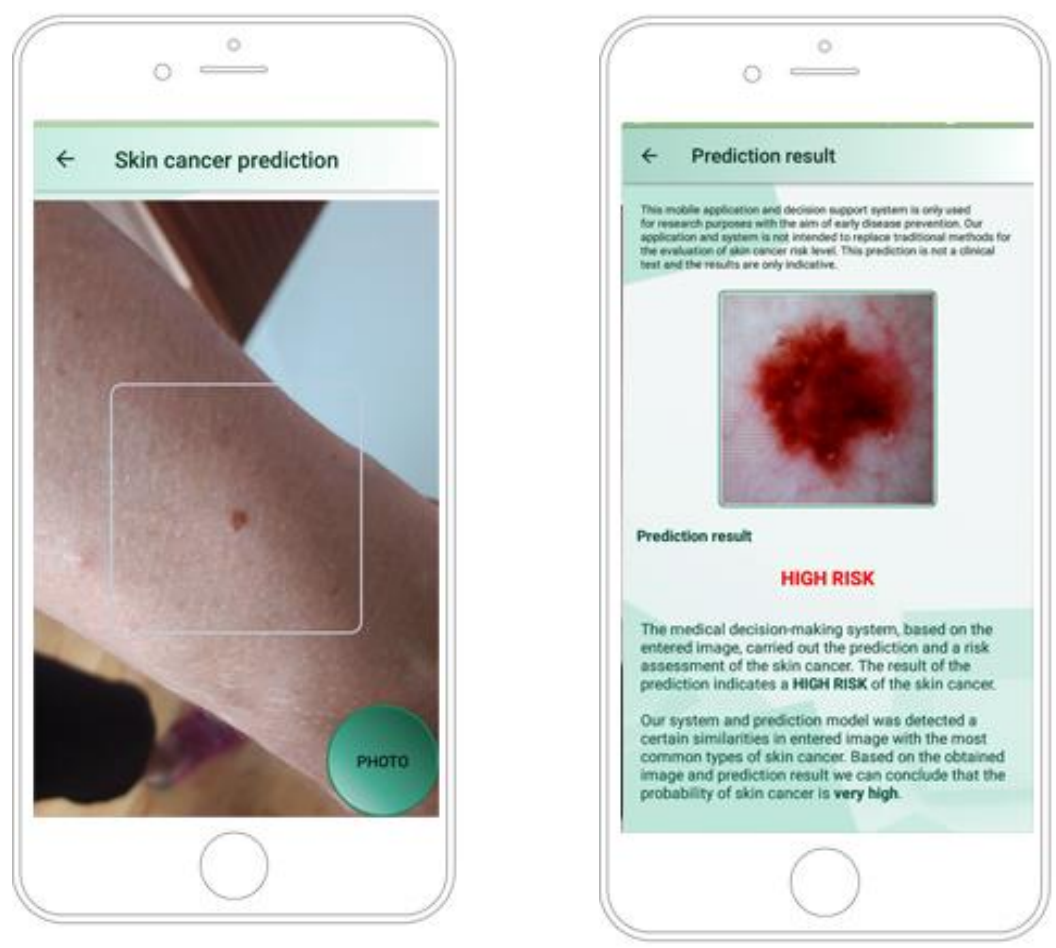

Fig. 4. Mobile application

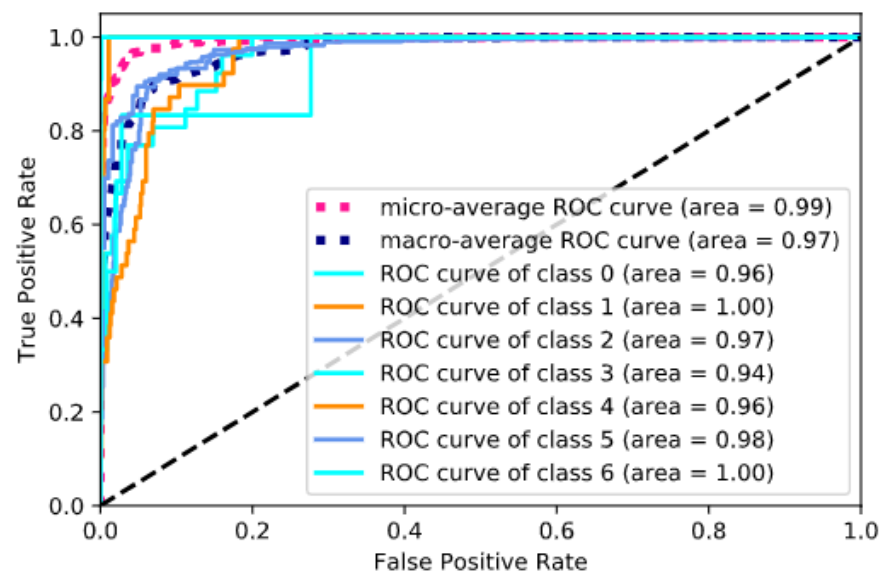

Fig. 5. ROC curves and classification accuracy 


\begin{tabular}{|c|c|c|c|}
\hline Reference Authors & Accuracy (\%) & Sensitivity (\%) & Specificity (\%) \\
\hline$[21]$ & 79.61 & 83.19 & 74.71 \\
\hline$[14]$ & 90.32 & 85.84 & 93.97 \\
\hline$[22]$ & 87.81 & 88.89 & 84.23 \\
\hline$[12]$ & 94.20 & 97.83 & 90.74 \\
\hline The proposed study & 93.20 & 94.34 & 91.48 \\
\hline
\end{tabular}

Table 3. Comparison of classification results

\section{Conclusion}

This paper has introduced a mobile application for skin cancer risk prediction. The aim of this research was to develop a user friendly mobile application for early prevention of skin cancer. An application whose work will be based on published research results in world scientific databases in the field of medicine.

The mobile application uses a new embedded CNN model based on MobileNetV2 library. The proposed application has three major activities. In prediction activity, the prediction process is executed. In the paper the architecture of the CNN classification model is presented as well as how the application is conducting the prediction process. The study shows good results for the proposed concept of classification model.

During the test period the application has been downloaded more than 1,500 people worldwide. The positive comments and suggestions from users on how to upgrade the application indicate that the main initial goal of the project has been achieved.

This research will be followed by additional testing, in order to improve the prediction model. . If we have taken only a small step in the prevention of this disease with this application, our goal has been fully achieved.

\section{References}

[1] World Health organication. [Online]. Available: https://www.who.int/uv/faq/skincancer/en/index1.html, accessed Jan. 2020.

[2] Georgescu, O. F. (2010)."Determining the Solution Space Form for an Indeterminate Pattern Recognition Problem: The "Skin Detection" Case.", Annals of DAAAM for 2010 \& Proceedings of the 21st International DAAAM Symposium, Volume 21, No. 1, ISSN 1726-9679 ISBN 978-3-901509-73-5, Editor B. Katalinic, Published by DAAAM International, Vienna, Austria 2010

[3] Jain, R.; Jain N. \& Aggarwal A. (2019). "Convolutional neural network based Alzheimer's disease classification from magnetic resonance brain images.", Cognitive System Reaserch, Vol. 57, pp. 147-159, DOI: 10.1016/j.cogsys.2018.12.015

[4] Khan S. U.; Islam N.; Jan Z.; Din I. U. \& Rodrigues J. J. P. C. (2019). "A novel deep learning based framework for the detection and classification of breast cancer using transfer learning.", Pattern Recognition Letters, Vol. 125, pp. 1-6, DOI: 10.1016/j.patrec.2019.03.022

[5] Chen S.; Ding C. \& Liu M.(2019). "Dual-force convolutional neural networks for accurate brain tumor segmentation.", Pattern Recognition, Vol. 88, pp. 90-100, DOI: 10.1016/j.patcog.2018.11.009

[6] Bui T.D.; Lee J.J. \& Shin J.(2019). "Incorporated region detection and classification using deep convolutional networks for bone age assessment." Artificial Intelligence in Medicine, Vol. 97, pp. 1-8, DOI: 10.1016/j.artmed.2019.04.005

[7] Bernal J.; Kushibar K.; Asfaw D. S.; Valverde S.; Oliver A.; Martí R. \& Lladó X.(2019). "Deep convolutional neural networks for brain image analysis on magnetic resonance imaging: a review.", Artificial Intelligence in Medicine, Vol. 95, pp. 64-81, DOI: 10.1016/j.artmed.2018.08.008

[8] Bourouis A.; Zerdazi A.; Feham M. \& Bouchachia A.(2013). "M-Health: Skin Ddsease analysis system using smartphone's camera.", Procedia Computer Science, Vol. 19, pp 1116-1120, DOI: 10.1016/j.procs.2013.06.157

[9] Brinker T.J.; Hekler A.; Enk A. H.; Klode J.; Hauschild A.; Berking C.; Schilling B.; Haferkamp S.; Schadendorf D.; Frohling S.; Utikal J. S.; von Kalle C. \& Collaborators (2019)."A convolutional neural network trained with dermoscopic images performed on par with 145 dermatologists in a clinical melanoma image classification task.", European Jurnal of Cancer, Vol. 111, pp. 148-154, DOI: 10.1016/j.ejca.2019.02.005

[10] Mahboda A.; Schaefer G.; Ellinger I.; Ecker R.; Pitiot A. \& Wang C.(2019). "Fusing fine-tuned deep features for skin lesion classification.", Computerized Medical Imaging and Graphics, Vol. 71, pp. 19-29, DOI: 10.1016/j.compmedimag.2018.10.007

[11] Harangi B.(2018). "Skin lesion classification with ensembles of deep convolutional neural networks.", Journal of Biomedical Informatics, Vol. 86, pp. 25-32, DOI: 10.1016/j.jbi.2018.08.006

[12] Abuzaghleh O.; Faezipour M. \& Barkana B. D.(2014). "Skincure: An innovative smart phone-based application to assist in melanoma early detection and prevention.", Signal \& Image Processing : An International Journal, Vol. 5, no. 6, pp. 1-15, DOI: $10.5121 /$ sipij.2014.5601 
[13] Phillips K.; Fosu O. \& Jouny I.(2015). "Mobile melanoma detection application for Android smart phones.", Biomedical Engineering Conf. (NEBEC), April 2015, pp. 1-2, DOI: 10.1109/NEBEC.2015.7117184

[14] Pal K.K. \& Sudeep K. S.(2016). "Preprocessing for image classification by convolutional neural networks.", Conference: 2016 IEEE International Conference on Recent Trends in Electronics, Information \& Communication Technology (RTEICT), May 2016, pp. 20-21, DOI: 10.1109/RTEICT.2016.7808140

[15] Sandler M.; Howard A.; Zhu M.; Zhmoginov A. \& Chen L.C.(2017). "MobileNetV2: Inverted Residuals and Linear Bottlenecks.", The IEEE Conference on Computer Vision and Pattern Recognition (CVPR), 2018, pp. 4510-4520

[16] Yim J. \& Sohn K.A.(2017). "Enhancing the performance of Convolutional Neural Networks on quality degraded datasets.", The International Conference on Digital Image Computing: Techniques and Applications (DICTA), Oct. 2017, pp. 1-8, DOI: 10.1109/DICTA.2017.8227427

[17] Namatēvs I.(2017). "Deep Convolutional Neural Networks: Structure, feature extraction and training.", Information Technology and Management Science, Vol. vol. 20, pp. 40-47, DOI: 10.1515/itms-2017-0007

[18] Trstenjak B.; Donko D. \& Avdagic Z.(2016). "Adaptable web prediction framework for disease prediction based on the hybrid Case Based Reasoning model.", Engineering, Technology \& Applied Science Research, Vol. 6, No. 6, pp. 1212-1216, ISSN: 1792-8036

[19] Kulhalli R.; Savadikar C. \& Garware B.(2019). "A hierarchical approach to skin lesion classification.", CoDSCOMAD '19: Proceedings of the ACM India Joint International Conference on Data Science and Management of Data, Jan. 2019, pp. 245-250, DOI: 10.1145/3297001.3297033

[20] Bernard S.; Chatelain C.; Adama S. \& Sabourin R.(2015). "The multiclass ROC front method for cost-sensitive classification.", Pattern Recognition, Vol. 52, pp. 46-60, DOI: 10.1016/j.patcog.2015.10.010

[21] Cavalcanti Pablo G. \& Scharcanski J.(2011). "Automated prescreening of pigmented skin lesions using standard cameras.", Computerized Medical Imaging and Graphics, Vol. 35, No. 6, pp. 481-491, DOI: 10.1016/j.compmedimag.2011.02.007

[22] Pandey A.; Sharma A. \& Ibrahim S.P.S.(2019). "Clinical image analysis for detection of skin cancer using Convolution Neural Networks.", Theranostics of Respiratory \& Skin Diseases (TRSD), May 2019, pp. 61-64, DOI: 10.32474/TRSD.2019.01.000111 